\title{
Clinical outcomes and complications of fiberoptic bronchoscopy in HIV-infected patients
}

\author{
Majid Marjani ${ }^{1}$, Mahtab Moinpour ${ }^{1}$, Arda Kiani ${ }^{2}$, Makan Sadr ${ }^{3}$, Habib Emami ${ }^{4}$, Roya Sepehrnia ${ }^{1}$, \\ Afshin Moniri ${ }^{3}$, Payam Tabarsi ${ }^{1}$, Davoud Mansouri ${ }^{1}$
}

${ }^{1}$ Clinical Tuberculosis and Epidemiology Research Center, National Research Institute of Tuberculosis and Lung Diseases (NRITLD), Shahid Beheshti University of Medical Sciences, Tehran, Iran

${ }^{2}$ Tracheal Diseases Research Center, National Research Institute of Tuberculosis and Lung Diseases (NRITLD), Shahid Beheshti University of Medical Sciences, Tehran, Iran

${ }^{3}$ Virology Research Center, National Research Institute of Tuberculosis and Lung Diseases (NRITLD), Shahid Beheshti University of Medical Sciences, Tehran, Iran

${ }^{4}$ Tobacco Prevention and Control Research Center, National Research Institute of Tuberculosis and Lung Diseases (NRITLD), Shahid Beheshti University of Medical Sciences, Tehran, Iran

\begin{abstract}
Introduction: Respiratory diseases, including infectious and non-infectious complications are common among patients with human immunodeficiency virus (HIV) infection. We aimed to determine the performance of fiberoptic bronchoscopy (FOB), bronchoalveolar lavage, and transbronchial lung biopsy in HIV-infected patients.

Material and methods: The current retrospective study was performed in Masih Daneshvari Hospital, Tehran, Iran, from January 2003 to March 2017. Patients with HIV infection and 836 episodes of respiratory manifestations were selected. Indications, outcomes as well as results and complications of bronchoscopy were extracted. Performance of bronchoscopy to determine definite etiology and its ability to rule out other differential diagnoses were evaluated.

Results: The indications of FOB were found in 289 subjects (34.6\%) with respiratory diseases, of whom only 220 patients had undergone the procedure. Bronchoscopy confirming a final diagnosis was found in 87 (39.5\%) patients and a diagnostic benefit was noted for minimum $66.7 \%$ of the subjects. Diagnostic ability of bronchoscopy was higher in patients suspected of pneumocystis jiroveci pneumonia (PJP), tuberculosis (TB), cytomegalovirus (CMV) pneumonitis, and in cases with ground-glass opacity and diffuse lung infiltrates ( $p$-value: 0.003 and 0.035 , respectively), and lower for subjects demonstrating reticulonodular infiltrations and right middle and lower lobes involvement ( $p$-value: 0.008, 0.005 , and 0.045 , respectively). Procedure-related complications were reported in five subjects.

Conclusions: FOB is a significantly advantageous and safe procedure in HIV-infected patients. Its diagnostic yield is higher in patients with ground-glass opacity and diffuse infiltration in chest computed tomography scan.
\end{abstract}

Key words: HIV, AIDS, pulmonary infection, bronchoscopy, bronchoalveolar lavage.

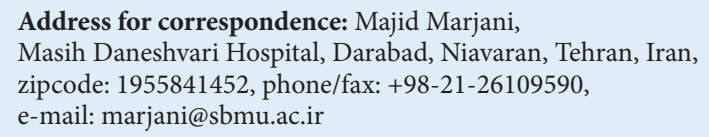

Article history:

Received: 03.05.2020

Received in revised form: 09.05 .2020

Accepted: 17.05.2020

Available online: 15.08 .2020
International Journal of HIV-Related Problems

HIV \& AIDS

R e v i e w 


\section{Introduction}

Since the beginning of the human immunodeficiency virus (HIV) epidemic, pulmonary complications were a significant cause of morbidity and mortality among HIV-infected patients [1] to such an extent that about $70 \%$ of them developed pulmonary complications in the course of the disease [2]. The spectrum of pulmonary manifestations is broad in patients infected with HIV, including many infectious and non-infectious complications [1]. Similar to other immunocompromised patients, prompt and accurate detection of the etiology of pulmonary infiltrates is essential for accurate and specific treatment [3]. A range of non-invasive and invasive methods are proposed for this purpose, but the optimal diagnostic strategy remains controversial [3, 4]. Fiberoptic bronchoscopy $(\mathrm{FOB})$ is a frequently used procedure for diagnosis and treatment in respiratory medicine. This procedure has progressively evolved and expanded, since its first introduction in $1968[5,6]$. The yield of FOB and related procedures, such as bronchoalveolar lavage (BAL) and transbronchial lung biopsy (TBLB) to diagnose HIV-associated opportunistic pulmonary diseases, was evaluated in various previous studies [7-14]. Most of these studies were conducted in the first decade after beginning of the HIV epidemic in Western countries, with different inclusion and exclusion criteria.

On the other hand, many factors affect the epidemiology of pulmonary diseases in HIV-infected patients, including geographical distribution of infectious agents, effective antiretroviral therapy, preventive measures etc. [1]. Therefore, due to high-rate of acquired immunodeficiency syndrome (AIDS) patients in some developing countries, re-evaluation of diagnostic utility of FOB in different settings is necessary [15].

The present study aimed at assessing the performance of FOB, BAL, and TBLB in detecting the etiology of pulmonary diseases in HIV-infected patients.

\section{Material and methods}

\section{Study population}

The current retrospective study was performed in Masih Daneshvari Hospital, National Research Institute of Tuberculosis and Lung diseases (NRITLD), Tehran, Iran, from January 2003 to March 2017. All HIV-infected patients admitted to the hospital in this period were recruited. Datasheet records and hospital information system were reviewed, and patients with respiratory complications were selected. Any admission due to respiratory complications was considered as one "episode". Re-admission during known pulmonary problem as well as second or more admissions were not counted. Based on the results of laboratory studies, consisting of microbiological investigations, acid-fast staining, molecular investigations for mycobacteria, and pleural fluid analysis as well as the results of lung imaging, the patients were deemed appropriate candidates for diagnostic FOB.

Demographic characteristics, bacteriological and pathological results related to samples obtained by FOB, contrain- dications for biopsy and FOB as well as FOB complications were recorded.

\section{Definitions}

These criteria were used to classify for final diagnosis as 'definite' or 'probable' in the current study:

Community-acquired pneumonia (CAP) and lung abscess were definite when blood or BAL culture (greater than $10^{4} \mathrm{CFU} / \mathrm{ml}$ ) [16] was positive with compatible symptoms and imaging. Also, ruling out tuberculosis was necessary.

Pulmonary tuberculosis (PTB) was confirmed by mycobacterial studies. Diagnosis of PTB in patients with granulomatous reaction in the lung tissue was confirmed when other causes were ruled out.

To confirm pulmonary infections with non-tuberculous mycobacteria (NTM), the last clinical practice guideline of the American Thoracic Society (ATS) was used [17].

Pneumocystis jiroveci pneumonia (PJP) was confirmed based on the presence of $P$. jiroveci in BAL or lung biopsy specimens using special staining or immunohistochemistry (IHC) assays.

Pulmonary aspergillosis was confirmed if: 1. Invasive fungal elements were found in lung tissue; 2. BAL fluid galactomannan index was $\geq 1$.

Cytomegalovirus (CMV) pneumonitis, anthracosis, Kaposi sarcoma, interstitial lung diseases, and organizing pneumonia were confirmed by pathological studies.

\section{Bronchoscopy technique}

Bronchoscopy was performed under general anesthesia with an injection of $0.02-0.05 \mathrm{mg} / \mathrm{kg}$ midazolam and $0.5 \mathrm{mg} / \mathrm{kg}$ propofol. Transoral FOB was conducted using an Olympus BFIT 240, after topical instillation of $2 \mathrm{ml}$ lidocaine $1 \%$ in the vocal cord and subglottic area. Normal saline in 20-50 ml aliquots was used to perform BAL. At least $40 \mathrm{ml}$ of instilled fluid was retrieved. TBLB was performed using Olympus cup forceps. All the patients were monitored for pneumothorax, hemorrhage, and hypoxemia in recovery room for at least two hours after the procedure.

\section{Performance of fiberoptic bronchoscopy}

Performance of bronchoscopy to detect etiology of pulmonary complications was calculated by proportion of patients with confirmatory FOB to the number of patients with definite diagnosis for their respiratory problem. Also, FOB ability to exclude other differential diagnoses was determined. The potential effects of HIV situation and treatment, radiographic images of the disease, ESR levels, and CD4 lymphocyte counts on the performance of bronchoscopy were evaluated.

\section{Ethical consideration}

The Ethics Committee of NRITLD approved the current study protocol. The research group committed to protect the privacy of all included patients. 


\section{Statistical analysis}

All data were entered into SPSS version 15.0 for statistical analysis. Descriptive statistics were employed to express qualitative variables as frequency and quantitative statistics to show quantitative variables as mean and standard deviation. To investigate the correlation between qualitative variables, $\chi^{2}$ test was applied. In order to show independent effect of determinants on dependent variables, such as diagnostic ability of FOB, logistic regression model was applied. $P$-value was considered as $\leq 0.05$.

\section{Results}

In the period of study, 1,416 admissions were recorded for 710 cases infected with HIV. Among them, 836 episodes of respiratory complications were observed. Mean and median of CD4 lymphocyte cell count were $90 \pm 131 \times 10^{6}$ and $40 \times 10^{6}$ cells/l, respectively. Thirty-one percent of patients with pulmonary complications were new cases of HIV infection, and $65.6 \%$ were ART-naïve.

In 289 admissions (34.6\% of all respiratory complications), fiberoptic bronchoscopy was indicated for a diagnosis approach. Finally, FOB was performed in 220 admissions. Mean age of patients was 38.2 \pm 9.7 years old (range, 9-66 years old). CD4 lymphocyte cell count ranged from 1 to $880 \times 10^{6}$ cells $/$, with the mean of $76 \pm 120 \times 10^{6}$ cells/l and the median of $30 \times 10^{6}$ cells/l. Demographic characteristics of patients are summarized in Table 1. Table 2 shows the reasons for not performing bronchoscopy for 69 patients, despite medical indications.

\section{Overall performance of fiberoptic bronchoscopy}

Definite final diagnosis was established for 135 patients, $61.4 \%$ of all 220 pulmonary cases complicated by HIV infection. FOB was diagnostic in 87 (39.5\%) episodes and successfully excluded other differential diagnosis in 135 cases (61.4\%). After combining both abilities, FOB was useful for 147 patients $(66.8 \%)$, while in 73 cases $(33.2 \%)$, FOB did not support confirmation of definite diagnosis or rejection of other causes.

No statistically significant correlation with the diagnostic ability of FOB were observed in the duration of HIV diagnosis (new vs. known cases), situation of antiretroviral therapy, CD4 lymphocyte cell count, and ESR level.

\section{Correlation between radiology and performance of fiberoptic bronchoscopy}

Concerning the type and site of pulmonary involvement, the diagnostic ability of FOB was higher for patients with ground-glass opacity and diffuse infiltration compared to other patients ( $p$-value: 0.003 and 0.035 , respectively). On the other hand, FOB was less effective for patients with reticulonodular infiltration, involvement of right middle lobe
Table 1. Characteristics of 220 episodes of pulmonary complications, for which fiberoptic bronchoscopy was performed

\begin{tabular}{l|c}
\hline Parameter & $n(\%)$ \\
\hline Age (years), mean \pm SD & $38.2 \pm 9.7$ \\
\hline Sex & $184(83.6)$ \\
\hline Male & $36(16.4)$ \\
\hline Female
\end{tabular}

\begin{tabular}{l|c}
\hline \multicolumn{2}{l}{\begin{tabular}{l} 
Risk factor for HIV infection* \\
\hline IDU
\end{tabular}} \\
\hline Sexual contact & $143(65.0)$ \\
\hline Prison & $19(44.1)$ \\
\hline Tattooing & $33(15.6)$ \\
\hline Others & $14(6.4)$ \\
\hline Unknown & $44(20.0)$ \\
\hline CD4 cell count & \\
\hline Mean $( \pm$ SD) & $76 \pm 120$ \\
\hline$<50$ & $114(63.3)$ \\
\hline $50-200$ & $49(27.2)$ \\
\hline $201-350$ & $12(6.7)$ \\
\hline$>350$ & $5(2.8)$
\end{tabular}

\begin{tabular}{l|c}
\hline HIV diagnosis $^{\#}$ & \multicolumn{1}{l}{} \\
\hline New cases & $78(36.1)$ \\
\hline Known cases & $138(63.9)$ \\
\hline ART situation ${ }^{\circledR}$ & $138(65.7)$ \\
\hline ART-naïve & $43(20.4)$ \\
\hline Under ART & $29(13.8)$ \\
\hline Drug interruption
\end{tabular}

${ }^{\star}$ Many cases had more than one risk factor for HIV infection. ${ }^{5} \mathrm{CD} 4$ count was available in 180 episodes. "Missing data: 4. ${ }^{\oplus M i s s i n g ~ d a t a: ~ 10 . ~ H I V ~-~ h u m a n ~}$ immune deficiency virus, IDU - intravenous drug user, ART - antiretroviral therapy

Table 2. Reasons for not performing bronchoscopy in 69 cases among 289 HIV-infected patients with pulmonary manifestations

\begin{tabular}{l|c|c|c}
\hline Reasons & $n$ & $\begin{array}{c}\% \\
\text { of total* }\end{array}$ & $\begin{array}{c}\% \\
\text { of group }\end{array}$ \\
\hline Not requested by physician & 12 & 4.1 & - \\
\hline Patient refusal & 18 & 6.2 & - \\
\hline \begin{tabular}{l} 
Medical reasons \\
\hline Cardiac problems
\end{tabular} & 39 & 13.4 & \\
\hline Severe hypoxemia & 5 & 1.7 & 10.3 \\
\hline $\begin{array}{l}\text { Positive sputum } \\
\text { smear for AFB }\end{array}$ & 2 & 0.7 & 5.1 \\
\hline $\begin{array}{l}\text { Intubation } \\
\text { Death before procedure }\end{array}$ & 5 & 1.7 & 12.8 \\
\hline
\end{tabular}

*All 289 HIV cases with indication of bronchoscopy. ${ }^{*}$ All 39 cases with medical reasons. HIV - human immune deficiency virus, AFB - acid fast bacilli 
Table 3. Correlation between radiology and diagnostic fiberoptic bronchoscopy ability among 220 HIV-positive cases

\begin{tabular}{|c|c|c|c|}
\hline $\begin{array}{l}\text { Radiologic } \\
\text { pattern }\end{array}$ & $\begin{array}{c}\text { Diagnostic FOB } \\
n(\%)^{*}\end{array}$ & $p$-value & $\begin{array}{c}\text { Odds ratio } \\
(95 \% \mathrm{Cl})\end{array}$ \\
\hline \multicolumn{4}{|c|}{ Lobar infiltration } \\
\hline No & $10(28.6)$ & \multirow{2}{*}{ NS } & \\
\hline Yes & 75 (41.7) & & \\
\hline
\end{tabular}

\begin{tabular}{|c|c|c|}
\hline Multilob & & \\
\hline No & $6(28.6)$ & \\
\hline Yes & $79(40.7)$ & Jive \\
\hline Cavitary & & \\
\hline No & $10(37.0)$ & NS \\
\hline Yes & 75 (39.9) & \\
\hline Lung ma & & \\
\hline No & $1(25.0)$ & \\
\hline Yes & $84(39.8)$ & TV \\
\hline
\end{tabular}

\begin{tabular}{|c|c|c|c|}
\hline \multicolumn{4}{|c|}{ Reticulonodular } \\
\hline No & $22(27.8)$ & \multirow{2}{*}{0.008} & \multirow{2}{*}{$\begin{array}{c}0.447 \\
(0.246-0.812)\end{array}$} \\
\hline Yes & $63(46.3)$ & & \\
\hline \multicolumn{4}{|c|}{ Milliary pattern } \\
\hline No & $2(66.7)$ & \multirow{2}{*}{ NS } & \\
\hline Yes & $83(39.2)$ & & \\
\hline \multicolumn{4}{|c|}{ Ground-glass opacity } \\
\hline No & $50(50.0)$ & \multirow{2}{*}{0.003} & \multirow{2}{*}{$\begin{array}{c}2.286 \\
(1.308-3.994)\end{array}$} \\
\hline Yes & $35(30.4)$ & & \\
\hline
\end{tabular}

Site of involvement
Left upper lobe
\begin{tabular}{l|l|l|l}
\hline No & $13(41.9)$ & \multirow{2}{*}{ NS } & \\
\hline Yes & $72(39.1)$ & & \\
\hline
\end{tabular}

\begin{tabular}{|c|c|c|}
\hline \multicolumn{3}{|l|}{ Lingula } \\
\hline No & $7(31.8)$ & \multirow{2}{*}{ NS } \\
\hline Yes & 78 (40.4) & \\
\hline \multicolumn{3}{|c|}{ Left lower lobe } \\
\hline No & $24(36.4)$ & \multirow{2}{*}{ NS } \\
\hline Yes & $61(40.9)$ & \\
\hline
\end{tabular}

\begin{tabular}{|c|c|c|c|}
\hline \multicolumn{4}{|c|}{ Right upper lobe } \\
\hline No & $13(38.2)$ & \multirow{2}{*}{ NS } & \\
\hline Yes & $72(39.8)$ & & \\
\hline \multicolumn{4}{|c|}{ Right middle lobe } \\
\hline No & $10(21.7)$ & \multirow{2}{*}{0.005} & \multirow{2}{*}{$\begin{array}{c}0.348 \\
(0.162-0.747)\end{array}$} \\
\hline Yes & $75(44.4)$ & & \\
\hline \multicolumn{4}{|c|}{ Right lower lobe } \\
\hline No & $13(27.1)$ & \multirow{2}{*}{0.045} & \multirow{2}{*}{$\begin{array}{c}0.490 \\
(0.242-0.993)\end{array}$} \\
\hline Yes & $72(43.1)$ & & \\
\hline \multicolumn{4}{|l|}{ Diffuse } \\
\hline No & $41(48.2)$ & \multirow{2}{*}{0.035} & \multirow{2}{*}{$\begin{array}{c}1.821 \\
(1.041-3.187)\end{array}$} \\
\hline Yes & $44(33.8)$ & & \\
\hline
\end{tabular}

*Details of imaging pattern was not available for 2 cases. FOB - fiberoptic bronchoscopy, HIV - human immunodeficiency virus, $\mathrm{Cl}$ - confidence interval, NS - no significant and right lower lobe compared to other patients ( $p$-value: $0.008,0.005$, and 0.045 , respectively). Table 3 demonstrates a correlation between radiology and bronchoscopy ability to determine the diagnosis.

\section{Performance of fiberoptic bronchoscopy in confirming specific diagnosis}

The most common causes of final diagnosis included PJP, tuberculosis, CAP, and CMV pneumonitis. Concerning PJP, diagnosis was confirmed by FOB in 43 of 81 cases who were suspected of PJP. In 22 cases, pneumocystis was found in BAL specimens, of which lung biopsy was performed in 17 of cases; all other were PJP consistent, except 1 patient. In 21 other cases, only lung biopsy was confirmative, although the results of BAL specimens for thirteen cases were not observed, but eight patients with confirmatory pathologic studies had negative IHC for pneumocystis in BAL specimens (one of them was diagnosed by TBLB in the second bronchoscopy).

Among 41 confirmed cases of tuberculosis, confirmation of diagnosis was made by FOB in 29 patients (70.7\%); 21 episodes were evaluated with positive results of $\mathrm{Myco}$ bacterium tuberculosis from BAL, 4 cases from biopsy samples (granuloma and other causes were excluded), and 4 positive cases were assessed based on post-bronchoscopy sputum.

Although, the aim of bronchoscopy was the exclusion of other causes in the setting of atypical presentation, bacteriological study of BAL specimens was positive in 11 cases revealing community acquired pneumonia, giving $55 \%$ of all and $64.7 \%$ of bacteriologically confirmed cases. Moreover, 11 proved cases of CMV pneumonitis were confirmed by bronchoscopy and histological examination.

Concerning non-infectious etiologies of pulmonary complications, 2 cases of organizing pneumonia was supported by FOB. For two patients, the first with lung fibrosis and the second with tracheoesophageal fistula, fiberoptic bronchoscopy was unproductive, and a confirmation was made during open-lung biopsy and rigid bronchoscopy, respectively. Table 4 shows the performance of FOB in confirmation of specific diagnosis in detail.

\section{Pathologic studies}

Biopsy of tissue specimen (lung biopsy) was conducted for 98 patients during bronchoscopy. Thirteen of 220 cases had a contraindication for bronchoscopic lung biopsy. Seven of them had a profound thrombocytopenia (less than $\left.50 \times 10^{9} / 1\right)$, three patients were severely hypoxemic, and three patients presented severe pulmonary hypertension. Pathologic studies were diagnostic for 52 (53\% of all) biopsy specimens. Lung tissue biopsy was performed in 24 cases suspected of TB, seven of them showed granuloma $(24.1 \%$ of confirmed cases of TB). Table 5 shows details of pathological results. 
Table 4. Yield of fiberoptic bronchoscopy to confirm final diagnosis, classified by specific causes

\begin{tabular}{l} 
Diagnosis \\
\cline { 5 - 6 }
\end{tabular}

*Sum of possible and definite cases. ${ }^{\beta}$ Kaposi sarcoma confirmed by skin biopsy, after excluding other causes of pulmonary infiltration considered as Kaposi sarcoma involvement, but lung biopsy was inconclusive. "Confirmed by open-lung biopsy. ${ }^{*}$ Confirmed by rigid bronchoscopy. FOB - fiberoptic bronchoscopy, CAP-community acquired pneumonia, PJP - pneumocystis jiroveci pneumonia, NTM - non-tuberculosis mycobacteria, CMV - cytomegalovirus, ILD - interstitial lung diseases

\section{Repeated bronchoscopy}

For 4 patients, FOB was repeated during the same episode of admission. The purpose of doing the second FOB was taking biopsy specimens from lung tissue. Two of these specimens were diagnostic, including one PJP and another PJP with concomitant CMV pneumonitis. For two other cases, a repetition of FOB did not increase diagnostic yield.

\section{Complications}

During 224 FOB procedures, there were 5 episodes of complications, including 3 incidents of pneumothorax, and 2 episodes of bleeding. One episode of bleeding led to intubation and finally to death. Age, radiologic pattern, or site of pulmonary involvement, and CD4 lymphocyte cell count did not have any significant correlation with the incidence of complication related to FOB. Although, the rate of complications was higher in the procedures with lung biopsy than procedures with BAL alone (4.1\% in comparison with $0.8 \%)$, this difference was not statistically significant $(p=0.172)$.

\section{Diagnostic studies on specimens}

Table 6 shows yields of different diagnostic studies on samples taking by FOB.
Table 5. Pathologic evaluations of lung tissue samples obtained by fiberoptic bronchoscopy in 98 HIV-infected patients

\begin{tabular}{l|c}
\hline Pathologic diagnosis & $n(\%)$ \\
\hline Anthracosis & $4(4.1)$ \\
\hline Consistent with PJP & $29(29.5)$ \\
\hline Granulomatous reactions & $7(7.1)$ \\
\cline { 2 - 2 } Without necrosis & 6 \\
\hline With necrosis & 1 \\
\hline CMV cytopathic changes & $2(2.0)$ \\
\hline Organizing pneumonia & $1(1.0)$ \\
\hline Combined patterns & $9(9.2)$ \\
\hline PJP + CMV & 7 \\
\hline PJP + CMV + organizing pneumonia & 1 \\
\hline Granulomatous reaction + CMV & 1 \\
\hline Non-specific & $37(37.7)$ \\
\hline Suboptimal specimen & $9(9.2)$ \\
\hline Total & 98 \\
\hline
\end{tabular}

*Non-diagnostic patterns including inflammation, chronic inflammation, necrosis, etc. FOB - fiberoptic bronchoscopy, HIV - human immune deficiency virus, PJP-pneumocystis jiroveci pneumonia, CMV - cytomegalovirus 
Table 6. Yields of different diagnostic tests on samples taking by fiberoptic bronchoscopy among 220 episodes of pulmonary complications in HIV-infected patients

\begin{tabular}{l|c|c|c|c}
\hline Diagnostic tests & $n$ & Positive, $n$ (\%) & Clinically important, $n$ (\%) & Main route of diagnosis, $n(\%)^{*}$ \\
\hline Bacteriology of BAL & 183 & $35(19.1)$ & $33(94.2)$ & $15(42.8)$ \\
\hline Fungal culture of BAL & 181 & $7(3.9)$ & $2(28.5)$ & 0 \\
\hline BAL GM & 49 & $7(14.2)$ & $2(28.5)$ & $22(100.0)$ \\
\hline BAL IHC for PJP & 110 & $22(20.0)$ & $22(100.0)$ & $15(88.2)$ \\
\hline BAL smear for AFB & 213 & $17(8.0)$ & $17(100.0)$ & $6(37.5)$ \\
\hline BAL culture for M. TB & 174 & $16(8.7)$ & $16(100.0)$ & $1(14.3)$ \\
\hline BAL PCR for M. TB & 115 & $7(6.1)$ & $6(85.7)^{*}$ & $4(40.0)$ \\
\hline PBS for M. TB & 89 & $10(11.2)$ & $10(100.0)$ & 0 \\
\hline BAL PCR for CMV & 40 & $29(72.5)$ & $2(6.8)$ & $38(73.0)$ \\
\hline TBLB & 98 & $52(53.0)^{\#}$ & $52(100.0)$ & \\
\hline
\end{tabular}

*Percentage of positive results. ${ }^{*}$ One false positive result. "Other than non-specific or sub-optimal specimens. FOB - fiberoptic bronchoscopy, HIV-human immune deficiency virus, $B A L$ - bronchoalveolar lavage, GM - galactomannan, IHC - immunohistochemistry, PJP - pneumocystis jiroveci pneumonia, AFB - acid fast bacilli, M. TB - Mycobacterium tuberculosis, PCR - polymerase chain reaction, PBS - post-bronchoscopy sputum, CMV-cytomegalovirus, TBLB - transbronchial lung biopsy

\section{Discussion}

In the current retrospective study, bronchoscopy confirmed a final diagnosis in $39.5 \%$ of the patients undergoing the procedure. If its role in excluding other common probable etiologies is considered, it can be said that FOB had a diagnostic benefit for minimum $66.7 \%$ of the subjects. Previous studies demonstrated a wide range of diagnostic yield for bronchoscopy in HIV-infected patients from $38 \%$ to $82.4 \%$ in different settings $[10,11,13,14,18-20]$. Most of these studies were conducted in the first decade of HIV epidemic, in Western countries with different inclusion and exclusion criteria. Just few of them contained more than 100 patients $[14,18$, 20]. It is important to consider that the efficacy of FOB is not restricted to its ability to confirm diagnosis. In many cases, ruling out of some important differential diagnoses such as TB is minimally of equal importance. Majority of previous studies did not consider this aspect of FOB usefulness.

The current study evaluations indicated that although some patients were eligible candidates for bronchoscopy, the clinicians did not request it $(n=12)$ or some problems prevented them from it (Table 2 ). In 18 episodes, the patients refused to undergo the procedure. With respect to patient's right to freely choose and decide on health services, this rate may decrease if clinicians devote more time to clarify the process of FOB and its advantages in the management of pulmonary diseases. If pre-bronchoscopy sputum smears were positive for acid fast bacilli, but FOB was still necessary to rule out other probable concomitant causes, the procedure can be postponed for few days to decrease the risk of contamination. It is possible for some pulmonary infections such as PJP that the chance of microorganism isolation is not affected by few days of empirical therapy [21]. Also, bronchoscopy can be carried out by experts in the intensive care unit (ICU) for intubated patients with careful monitoring [22]. The risks and benefits of this procedure should be carefully considered in mechanicallyventilated patients [5].

Diagnostic utility of FOB was higher among patients with ground glass opacity and diffuse infiltrates in imaging studies. This relationship may be justified by higher likelihood of PJP among such patients and high ability of FOB to detect it $[23,24]$. There was no rational explanation for inefficacy of FOB in patients demonstrating reticulonodular infiltrates and right middle and lower lobes involvement.

Yield of bronchoscopy was not equal for different pulmonary diseases. Among infectious processes, patients with PJP, TB, CMV pneumonitis, and CAP (atypical cases with difficulty to differentiate them from TB) had higher gain from FOB. Kennedy et al., compared yield of bronchoscopy between HIV-infected and uninfected patients suspected of TB and did not find any differences [12]. They and other authors found that BAL can improve and accelerate the diagnosis of PTB in HIV-infected patients [12, 18, 25].

In the current study, lung biopsy was diagnostic among $24.1 \%$ of the TB confirmed cases. In one study, TBLB provided the highest yield for an earlier diagnosis of TB among all bronchoscopic specimens [26]. As pathologic sampling was not performed for all the subjects suspected of TB, its contribution to the diagnosis of TB cannot be justified.

Although polymerase chain reaction (PCR) for the detection of M. tuberculosis was performed on $115 \mathrm{BAL}$ specimens, it was the exclusive route of diagnosis in one patient only. Concerning high cost, little benefit, and possibility of false positive result, routine PCR for M. tuberculosis is not rational. It can be performed for samples containing acid fast bacilli in order to differentiate TB from NTM.

Previous studies showed high utility for BAL in the diagnosis of PJP $[9,14]$, and some of them recommended that TBLB should be reserved for selected cases and not performed routinely [9]. There were minimum eight patients, that despite IHC-negative BAL specimens were pathologi- 
cally confirmed for PJP. Also, eight patients had concomitant PJP and CMV, which the latter was exclusively diagnosed by a pathologic analysis. These two groups contained $37.2 \%$ of all confirmed cases of PJP. Based on these results, it may be sensible to recommend biopsy for every HIV-infected patient suspected of PJP.

CMV PCR result was positive in 29 BAL specimens (72.5\% of all specimens assessed for CMV), in which just two patients had CMV pneumonitis. To date, the importance of CMV in BAL specimens is not clear [1]. Due to high-rate of false positive results, it seems unnecessary as a routine test among HIV-infected patients.

Although some primary studies showed high frequency (up to 20\%) of complications, especially in HIV-infected patients undergoing TBLB [9, 27-29], the current study found bronchoscopy as a safe technique, with a $2.2 \%$ risk of complications and no significantly higher rate of morbidity among patients with lung biopsy. The findings of the present study were consistent with those showing safety of FOB and TBLB among HIV-infected patients [8, 13, 30, 31].

The evaluation of sputum for acid fast bacilli in consequent days after bronchoscopy (PBS) proved to be an effective method for early diagnosis of TB [32]. In the present study, $\mathrm{PBS}$ was the main route for $\mathrm{TB}$ diagnosis in $4.5 \%$ of the patients with $\mathrm{TB}$, while it was $12 \%$ and $7 \%$ in other studies [12,33], respectively, and the efficacy of PBS smears was not significantly related to HIV infection [32].

The current study had some limitations. First, it was retrospective in nature, with all challenges related to this kind of studies. A great effort was made to minimize missing information by a meticulous search in the existing data. Second, it was conducted in a referral center for TB. Therefore, the rate of TB cases may be higher than those of other settings. For better understanding of the role of bronchoscopy in pulmonary diseases of HIV-infected patients, large, multicenter, and preferably prospective studies are required. Such studies can evaluate diagnostic yield of newer assays, such as Genexpert TB test or biomarkers (for example, BAL galactomannan) as comparable to traditional tests.

\section{Conclusions}

The present study demonstrated that FOB is greatly helpful in HIV-infected patients. When non-invasive methods fail to diagnose the cause of pulmonary infiltration, bronchoscopy can confirm the final etiology and support to narrow differential diagnosis. Diagnostic utility of FOB is higher for HIV-infected patients suspected of PJP, TB, or CMV pneumonitis. Its efficacy is also high in patients with ground-glass opacity and diffuse infiltrations, but low in cases with right middle and lower lobes involvement. Overall, it is a safe procedure with a low rate of complications.

\section{Conflict of interest}

The authors declare no conflict of interest with respect to the research, authorship, and/or publication of this article.

\section{References}

1. Benito N, Moreno A, Miro JM, Torres A. Pulmonary infections in HIV-infected patients: an update in the 21st century. Eur Respir J 2012; 39: 730-745.

2. Miller R. HIV-associated respiratory diseases. Lancet 1996; 348 : 307-312.

3. Danes C, Gonzalez-Martin J, Pumarola T, et al. Pulmonary infiltrates in immunosuppressed patients: analysis of a diagnostic protocol. J Clin Microbiol 2002; 40: 2134-2140.

4. Crawford SW. Noninfectious lung disease in the immunocompromised host. Respiration 1999; 66: 385-395.

5. Du Rand IA, Blaikley J, Booton R, et al. British Thoracic Society guideline for diagnostic flexible bronchoscopy in adults: accredited by NICE. Thorax 2013; 68 Suppl 1: i1-i44.

6. Ikeda S, Yanai N, Ishikawa S. Flexible bronchofiberscope. Keio J Med 1968; 17: 1-16.

7. Pedersen U, Hansen IM, Bottzauw J. The diagnostic role of fiberoptic bronchoscopy in AIDS patients with suspected Pneumocystis carinii pneumonia. Arch Otorhinolaryngol 1989; 246: 362-364.

8. de Blic J, Blanche S, Danel C, Le Bourgeois M, Caniglia M, Scheinmann P. Bronchoalveolar lavage in HIV infected patients with interstitial pneumonitis. Arch Dis Child 1989; 64: 1246-1250.

9. Griffiths MH, Kocjan G, Miller RF, Godfrey-Faussett P. Diagnosis of pulmonary disease in human immunodeficiency virus infection: role of transbronchial biopsy and bronchoalveolar lavage. Thorax 1989; 44: 554-558.

10. Fischer B, Elmer M, Ramadori G, Lorenz J. HIV-associated infections: indications and importance of fiberoptic bronchoscopy. Pneumologie 1990; 44 Suppl 1: 316-317.

11. Heurlin N, Elvin K, Lidman C, Lidman K, Lundbergh P. Fiberoptic bronchoscopy and sputum examination for diagnosis of pulmonary disease in AIDS patients in Stockholm. Scand J Infect Dis 1990; 22: 659-664.

12. Kennedy DJ, Lewis WP, Barnes PF. Yield of bronchoscopy for the diagnosis of tuberculosis in patients with human immunodeficiency virus infection. Chest 1992; 102: 1040-1044.

13. Henriquez A, Srur E, Giglio M, et al. Etiologic diagnosis of pneumonia in HIV-AIDS patients. Usefulness of fibrobronchoscopy. Rev Med Chil 1993; 121: 279-285.

14. Steiner S, Schwalen A, Klein RM, et al. Results and complications of fiber bronchoscopy in HIV positive patients. Pneumologie 1998; 52: 694-699.

15. Ghosn J, Taiwo B, Seedat S, Autran B, Katlama C. HIV. Lancet 2018; 392: 685-697.

16. van der Eerden MM, Vlaspolder F, de Graaff CS, Groot T, Jansen HM, Boersma WG. Value of intensive diagnostic microbiological investigation in low- and high-risk patients with community-acquired pneumonia. Eur J Clin Microbiol Infect Dis 2005; 24: 241-249.

17. Griffith DE, Aksamit T, Brown-Elliott BA, et al. An official ATS/ IDSA statement: diagnosis, treatment, and prevention of nontuberculous mycobacterial diseases. Am J Respir Crit Care Med 2007; 175: 367-416.

18. Worodria W, Davis JL, Cattamanchi A, et al. Bronchoscopy is useful for diagnosing smear-negative tuberculosis in HIV-infected patients. Eur Respir J 2010; 36: 446-448.

19. Supervia Caparros A, Minguez Maso S, Galofre Alvaro N, Knobel Freud H, Currull Serrano V, Sanjuás Benito C. Fiber optics bronchoscopy yield and predictive clinical, analytical, and radiologic factors in the etiologic diagnosis of pulmonary pathology in patients with HIV infection. An Med Interna 1993; 10: 532-536.

20. Baughman RP, Dohn MN, Frame PT. The continuing utility of bronchoalveolar lavage to diagnose opportunistic infection in AIDS patients. Am J Med 1994; 97: 515-522.

21. O'Donnell WJ, Pieciak W, Chertow GM, Sanabria J, Lahive KC. Clearance of Pneumocystis carinii cysts in acute P. carinii pneumo- 
nia: assessment by serial sputum induction. Chest 1998; 114: 1264-

1268

22. Fecci L, Consigli GF. Bronchoscopy in intensive care unit. Monaldi Arch Chest Dis 2011; 75: 67-71.

23. Huang L, Hecht FM, Stansell JD, Montanti R, Hadley WK, Hopewell PC. Suspected Pneumocystis carinii pneumonia with a negative induced sputum examination. Is early bronchoscopy useful? Am J Respir Crit Care Med 1995; 151: 1866-1871.

24. Huang L, Cattamanchi A, Davis JL, et al. HIV-associated Pneumocystis pneumonia. Proc Am Thorac Soc 2011; 8: 294-300.

25. Santoso P, Soeroto AY, Juniati R, et al. Improving diagnostic of pulmonary tuberculosis in HIV patients by bronchoscopy: a cross sectional study. Acta Med Indones 2017; 49: 330-335.

26. Salzman SH, Schindel ML, Aranda CP, Smith RL, Lewis ML The role of bronchoscopy in the diagnosis of pulmonary tuberculosis in patients at risk for HIV infection. Chest 1992; 102: 143-146.

27. Coker RJ, Mitchell DM. The role of bronchoscopy in patients with HIV disease. Int J STD AIDS 1994; 5: 172-176.

28. Orenstein M, Webber CA, Cash M, Heurich AE. Value of bronchoalveolar lavage in the diagnosis of pulmonary infection in acquired immune deficiency syndrome. Thorax 1986; 41: 345-349.

29. Milligan SA, Luce JM, Golden J, Stulbarg M, Hopewell PC. Transbronchial biopsy without fluoroscopy in patients with diffuse roentgenographic infiltrates and the acquired immunodeficiency syndrome. Am Rev Respir Dis 1988; 137: 486-488.

30. Simpson FG, Arnold AG, Purvis A, Belfield PW, Muers MF, Cooke NJ Postal survey of bronchoscopic practice by physicians in the United Kingdom. Thorax 1986; 41: 311-317.

31. Cazzadori A, Di Perri G, Todeschini G, et al. Transbronchial biopsy in the diagnosis of pulmonary infiltrates in immunocompromised patients. Chest 1995; 107: 101-106.

32. Malekmohammad M, Marjani M, Tabarsi P, et al. Diagnostic yield of post-bronchoscopy sputum smear in pulmonary tuberculosis. Scand J Infect Dis 2012; 44: 369-373.

33. George PM, Mehta M, Dhariwal J, et al. Post-bronchoscopy sputum: improving the diagnostic yield in smear negative pulmonary TB. Respir Med 2011; 105: 1726-1731. 